\title{
LABOR DEL AUDITOR ANTE LA AUDITORÍA DE GESTIÓN
}

\author{
Dr. JULIO V. FLORES KONJA (*) \\ Lic. 'ANA MARIA GUTIÉRREZ HUBY (**) \\ "No nos falta valor para emprender ciertas cosas porque son \\ dificiles, sino que son dificiles porque nos falta valor para emprenderlas".
}

Séneca

Desde hace algunos años se está cuestionando, por parte de los usuarios, la auditoría financiera y contable, entendiéndose como tal aquella que tiene por finalidad expresar una opinión sobre la razonabilidad de los estados financieros.

La mayor crítica que ha recibido la labor de los auditores se basa en que su trabajo se realiza siempre sobre datos históricos y que su única conclusión es si los estados financieros son razonablemente correctos o no. Los directores se quejan de que este planteamiento tiene su utilidad en la mayoría de ocasiones para terceras personas ajenas a la entidad, como inversionistas, acreedores, administración, etc., pero ayuda muy poco a la dirección, preocupada por la gestión y el futuro desarrollo de la entidad.

El primer paso dado por los auditores para ofrecer a los directores de las entidades algo más constructivo que el solo informe de auditoría fue la aparición de la carta de recomendaciones o de sugerencias para la mejora de los procedimientos contables y administrativos de la entidad.
En muchas ocasiones esa carta se hace más con una visión comercial que con el rigor profesional con que debería hacerse.

La carta de recomendaciones no fue suficiente, el mercado pedía más y fueron apareciendo otra serie de servicios a los que la tendencia anteponía siempre la palabra auditoría; así surgió la auditoría operacional, la auditoría administrativa, la auditoría analítica, etc., aunque estas denominaciones no siempre definan actuaciones profesionales diferentes.

Todos estos nuevos servicios tienen un denominador común, revisan los procedimientos pero no colaboran de una manera directa, en la gestión de la entidad. Hasta ese momento podíamos agrupar los servicios que prestaban los auditores en dos grupos, auditoría financiera y auditoría de procedimientos, cuyas diferencias más importantes eran:

(*) Docente Principal y Director de la Escuela Académico Profesional de Contabilidad de la Facultad de Ciencias Contables de la UNMSM.

(**) Docente Asociado de la Facultad de Ciencias Contables de la UNMSM. 


\begin{tabular}{|l|l|l|}
\hline & AUDITORÍA FINANCIERA & $\begin{array}{l}\text { AUDITORÍA DE } \\
\text { PROCEDIMIENTOS }\end{array}$ \\
\hline Periodicidad & Anual & Eventual \\
\hline Objetivo & $\begin{array}{l}\text { Opinión sobre la razonabilidad } \\
\text { estados financieros. }\end{array}$ & $\begin{array}{l}\text { Evaluar procedimientos } \\
\text { y sugerir mejoras }\end{array}$ \\
\hline Normas para su realización & $\begin{array}{l}\text { Normas de auditoría generalmente } \\
\text { aceptadas }\end{array}$ & No existe \\
\hline $\begin{array}{l}\text { Ayuda a la dirección en la } \\
\text { gestión }\end{array}$ & Nada & Débilmente \\
\hline
\end{tabular}

El informe de auditoría no contiene toda aquella información que pudiera ser de interés para los directivos de la entidad. Únicamente en aquellos casos en que el principio de gestión continuada se ve comprometida el auditor se separa de la rigurosidad de las normas y procedimientos de auditoría para evaluar la continuidad o no de la entidad auditada.

Los auditores han huido siempre de sus relaciones con cualquier trabajo que tenga una fuerte carga subjetiva, a pesar de que su formación forjada por años de experiencia en todas las actividades del comercio y la industria les permitiría una mayor colaboración con las entidades auditadas.

En nuestra opinión, los auditores actuales están desaprovechados, su formación es superior a la necesaria para la realización de una auditoría financiera; además, sus clientes no saben aprovechar la capacidad de síntesis y de análisis de este profesional.

La tendencia actual, en relación con la información suministrada por las empresas, es incrementar su volumen en una mayor cantidad de datos referentes a la gestión y futuro de la entidad. Además de los fríos números de los estados financieros.
El inversionista está solicitando cada vez más información sobre la marcha de la entidad y su futuro, preferentemente las cifras históricas de los estados financieros; bajo el punto de vista del inversionista el planteamiento sería el informe de gestión y lo secundario las cifras anuales.

En Estados Unidos, la comisión de Valores de la Bolsa de Nueva York exigió, a partir del año 1980, que las Memorias de las sociedades incluyeran una manifestación sobre las bondades de los controles internos de la sociedad, indicando además el porqué de no establecer las recomendaciones realizadas por los auditores. Los auditores a su vez debían manifestar su conformidad o reparos con lo indicado por la dirección en dicha carta.

La Cuarta Directriz de la Comunidad . Económica Europea consideraba como parte integrante de las cuentas anuales el Informe de Gestión, y se configuraba así como un documento de obligada confección y difusión en el marco más amplio de la información que debían suministrar las sociedades europeas. 
El contenido del informe de gestión se agrupaba en cuatro puntos:

a) Los acontecimientos importantes acaecidos después del cierre del ejercicio.

b) La evolución previsible de la sociedad.

c) Las actividades en materia de investigación y desarrollo.

d) Las indicaciones necesarias en el caso de adquisición de acciones propias.

Es evidente que existe una tendencia a que las direcciones de las sociedades adquieran una mayor responsabilidad frente al público inversionista mediante el suministro de más información que permitirá evaluar mejor su gestión. Si por una necesidad obvia de información verazy objetiva se desarrolló la función de los auditores ¿qué impedirá que ese requisito de veracidad y objetividad sea solicitado para la gestión y la información de la gestión?

Es en este punto donde aparece la auditoría de gestión, entendiéndola como el análisis permanente de la gestión de una entidad o de parte de ella con la finalidad de emitir un juicio profesional.

Pensando en una entidad como en un todo formado por departamentos, donde cada uno de ellos realiza su propia gestión, la auditoría se puede realizar sobre la totalidad de la entidad o sobre cualquiera de sus departamentos. En el primer caso la podemos llamar Auditoría de la Gestión Gerencial; y en el segundo, dependiendo del departamento a auditar podremos hablar de auditoría de ventas, recursos humanos, etcétera.

Antes de entrar en lo que podríamos denominar una guía para la auditoría de gestión, deberíamos considerar hacer algunos matices. Si separásemos la gestión en tres momentos, la fase de captación de información, la de análisis y la toma de decisiones podremos diferenciar gráficamente la auditoría de gestión de los restantes tipos de auditoría.

Está claro que la auditoría financiera y la operativa o administrativa tienen como objeto el examen de la información o el proceso de esa información; mientras que la auditoría de gestión tiene por finalidad auditar las decisiones de la dirección, ya sea general o departamental; pero con posterioridad a la toma de decisiones ya que, en caso de hacerlo con anterioridad, se podría hablar de funciones de consultoría.

En este momento, se podría hacer una reflexión ¿es la formación del auditor actual la ideal para la realización de la auditoría de gestión? En nuestra opinión, en lo referente a la gestión financiera sin duda alguna; en el resto y en la gerencial necesitará una formación adicional en el campo de la alta dirección.

Se expone a continuación una guía con algunos de los puntos más importantes que pueden servir para evaluar la gestión de una entidad.

\begin{tabular}{|l|c|c|}
\hline INFORMACIÓN & ANÁLISIS & DECISIÓN \\
\hline $\begin{array}{l}\text { Auditoría } \\
\text { Financiera y } \\
\text { Administrativa }\end{array}$ & Auditoría de Gestión \\
\hline
\end{tabular}


Se ha dividido esta guía en cuatro partes:

- Compras

- Recursos Humanos

- Ventas

- Finanzas

\section{COMPRAS}

Objetivo:

Comprar en óptimas condiciones (calidad, precio y forma de pago).

Mantener la existencia necesaria para el proceso de fabricación o para la venta.

\section{Guía:}

Hacer un inventario de las compras por productos de los últimos tres años.

Hacer un inventario de las compras por proveedores de los últimos tres años.

Solicitar ofertas de los principaIes articulos para proveedores alternativos.

Estudiar la evolución de los precios de los principales productos.

Países (si sea el caso) de donde proceden las compras de importación.

Existencia media de los últimos tres años.

Rotación de almacén de materias primas en los últimos tres años.

Plazo medio de entrega de las principales materias primas.

VENTAS

Objetivo:

Vender en las mejores condiciones.
Cobrar la totalidad de lovendido.

Incrementar la participación en el mercado.

Guía:

Hacer un inventario de las ventas por productos en los últimos tres años.

Hacer un inventario de las ventas por clientes de los últimos tres años.

Obtener explicación de la dirección de las principales desviaciones habidas en los puntos anteriores.

Obtener las tarifas de precios de los últimos tres años.

Obtener la cifra de ventas de los últimos tres años.

Hacer un cuadro comparativo de las ventas anuales restándoles a cada año la incidencia del aumento de precios o calcular las ventas a precios constantes del primer año.

Obtener el nombre de los principales competidores.

Obtener los precios de ventas de los principales competidores. Analizar la evolución de los márgenes en los últimos tres años. Realizar una evolución de la morosidad de los últimos tres años.

Existencia media de los productos acabados.

Costo de ventas por año.

Rotación de los productos acabados.

Plazo medio de entrega.

\section{RECURSOS HUMANOS}

Objetivos:

Bienestar social.

Productividad. 
Guia:

Obtener una pirámide de edades.

- Obtener un detalle por años de antigüedad de toda la planilla.

Obtener el porcentaje de absentismo de los últimos años.

- Obtener el número de horas pérdidas por conflictos laborales.

- Si se dispone de convenio propio compararlo con el del sector.

- Número de horas dedicadas a la capacitación del personal.

- Número de accidentes laborales habidos y horas pérdidas.

FINANZAS

\section{Objetivos:}

Obtener la máxima rentabilidad de los capitales invertidos con el mínimo riesgo.

\section{Guía:}

Obtener los estados financieros de los últimos cinco años.

- Comparar y analizar las variaciones entre los principales capítulos del balance y estado de origen y aplicación de fondos.

Realizar un cuadro de mando con los principales ratios de los últimos años.

Obtener el plazo medio de cobro de los clientes.

- Obtener el plazo medio de pago de los proveedores.

Obtener el costo medio de la estructura financiera y compararlos con los del mercado actual.

- Obtener el grado de apalancamiento financiero de la sociedad.
- Analizar la política de dividendos de los últimos años.

Revisar los planes futuros e inversión de la sociedad y averiguar si se han hecho los oportunos estudios de inversión.

La Auditoría de Gestión que debería ofrecer actualmente el Auditor es el examen crítico de las decisiones tomadas en el seno de las empresas.

Es evidente que en el vertiginoso mundo actual de los negocios la información, la rapidez y la oportunidad en la toma de decisiones pueden marcar las pautas de los resultados futuros de un ente económico.

El auditor al intervenir en auditoría de gestión deberá examinar de forma constructiva las decisiones tomadas en su momento, no desde el punto de vista administrativo sino bajo el prisma de la objetividad de las decisiones tomadas. Generalmente, cualquier decisión importante se toma por el criterio personal aplicado sobre estudios realizados a partir de la información de que se dispone y ante la expectativa de obtención de unos resultados estimados.

El auditor examina todo el proceso de toma de decisiones, y si los resultados ya han sido obtenidos para averiguar, en el caso de que no se hayan obtenido los esperados, ¿cuáles han sido las circunstancias concurrentes que han provocado la desviación?

La problemática fundamental en este tipo de actuaciones es la que se deriva de la necesidad, por parte del auditor, de poseer un elevado conocimiento de la empresa y del sector en el que la misma se desenvuelve. 
Se ha dividido esta guía en cuatro partes:

- Compras

- Recursos Humanos

- Ventas

- Finanzas

\section{COMPRAS}

Objetivo:

Comprar en óptimas condiciones (calidad, precio y forma de pago).

Mantener la existencia necesaria para el proceso de fabricación o para la venta.

Guía:

Hacer un inventario de las compras por productos de los últimos tres años.

Hacer un inventario de las compras por proveedores de los últimos tres años.

Solicitar ofertas de los principales artículos para proveedores alternativos.

Estudiar la evolución de los precios de los principales productos.

Países (si sea el caso) de donde proceden las compras de importación.

Existencia media de los últimos tres años.

Rotación de almacén de materias primas en los últimos tres años.

Plazo medio de entrega de las principales materias primas.

VENTAS

Objetivo:

Vender en las mejores condiciones.
Cobrar la totalidad de lo vendido.

Incrementar la participación en el mercado.

Guía:

Hacer un inventario de las ventas por productos en los últimos tres años.

Hacer un inventario de las ventas por clientes de los últimos tres años.

Obtener explicación de la dirección de las principales desviaciones habidas en los puntos anteriores.

Obtener las tarifas de precios de los últimos tres años.

Obtener la cifra de ventas de los últimos tres años.

Hacer un cuadro comparativo de las ventas anuales restándoles a cada año la incidencia del aumento de precios o calcular las ventas a precios constantes del primer año.

Obtener el nombre de los principales competidores.

Obtener los precios de ventas de los principales competidores. Analizar la evolución de los márgenes en los últimos tres años. Realizar una evolución de la morosidad de los últimos tres años.

Existencia media de los productos acabados.

Costo de ventas por año.

Rotación de los productos acabados.

Plazo medio de entrega.

\section{RECURSOS HUMANOS}

Objetivos:

Bienestar social.

Productividad. 
El alto grado de responsabilidad de los auditores, la problemática derivada de la necesidad de conocer las tendencias de los distintos sectores empresariales y la conveniencia de la intervención de profesionales expertos en diversas áreas han hecho que este tipo de actuaciones se encuentren muy poco extendidas.

En la práctica, el Auditor interviene para analizar si la dirección de la empresa ha intentado obtener y estudiar objetivamente toda la información posible antes de proceder a la toma de decisiones.
COLEGIO DE CONTADORES DE PICHINCHA ECUADOR

Noviembre 2000.

http://www4.ccpp.org.ec.

1998. Manual de Auditoría de Gestión. Buenos Aires.

http://www.respondanet.com

NAVARROGOMOLLÓN, Ángel J.

1998. "Ética en las Relaciones EmpresaAuditor". En: Revista Alta Dirección. Año 32 (198). Marzo - Abril.

ALDOMA LA ROCA, Juan., GARRIGA OBIOLS, J. Miguel.

1989. "Trabajos que pueden realizar los Auditores". En: Revista Alta Dirección. Año 18 (101). Enero - Febrero. 